\title{
RATIONAL FOR THE USE OF COCONUT OIL-BASED ANTI-MYCOTIC PESSARIES TO COMBAT RECURRENT VAGINAL INFECTION: IN VITRO/IN VIVO EVALUATION AND PRELIMINARY PROSPECTIVE CLINICAL INVESTIGATION
}

\author{
RABAB KAMEL ${ }^{1,}{ }^{*}$, HAIDY ABBAS ${ }^{2}$ \\ ${ }^{1}$ Pharmaceutical Technology Department, National Research Centre, El-Bohooth Street, Cairo, Egypt, ${ }^{2}$ Pharmaceutics Department, Faculty \\ of Pharmacy, Damanhour University, Egypt \\ Email: drrababk@hotmail.com
}

Received: 28 Jun 2018, Revised and Accepted: 19 Jul 2018

\begin{abstract}
Objective: Coconut oil ( $\mathrm{CO}$ ) was used in combination with metronidazole in order to prepare vaginal pessaries ameliorating the use, delivery and efficacy of the anti-mycotic treatment.

Methods: To prepare the metronidazole-loaded pessaries, different lipids, namely: suppocire NA15 (SNA), suppocire NAIS10 (SIS), suppocire AM (SAM) and ovucire WL 2944 (OWL) were used alone or in combination with coconut oil in a ratio of 1:1. Prepared pessaries were subjected to characterization and evaluation of physical properties, drug release, anti-microbial effect, in vivo studies and histopathological examination.

Results: In the case of all the lipids, coconut oil improved the physical properties which can allow better use and performance of the pessaries. Among the prepared pessaries, the $\mathrm{OWL} / \mathrm{CO}$-based pessary (P8) showed the highest drug release profile $(\mathrm{P}<0.05)$. Characterization was done using $\mathrm{X}$-Ray diffraction. Microbiological study showed the superiority of OWL/CO-based pessaries over OWL-based pessaries at different time intervals. Histopathological studies of the vagina of female Wistar rats showed that in the case of the Candida albicans-infected group treated with the selected pessary, the mucosal and muscular layer returned to its normal structure, in addition to the disappearance of the cauliflower-like projections and the newly formed blood capillaries, seen in the Candida albicans-infected group. The questionnaire done in volunteers suffering from recurrent vaginal infection reported a significant decrease (reaching $93 \%$ ) in the severity of the symptoms, after the use of the suggested pessaries (P8) $(\mathrm{P}<0.05)$. Also, the suggested product had high patient acceptability. The results reported after the use of P8 were better than those of Amrizole ${ }^{\circledR}$ vaginal suppository.
\end{abstract}

Conclusion: The current study can present a successful, effective and easy to use product with low cost and minimal side effects.

Keywords: Coconut oil, Ovucire, Vaginal, Pessary, Microbiology

(C) 2018 The Authors. Published by Innovare Academic Sciences Pvt Ltd. This is an open access article under the CC BY license (http://creativecommons.org/licenses/by/4.0/) DOI: http://dx.doi.org/10.22159/ijap.2018v10i5.28199

\section{INTRODUCTION}

A large number of women suffer from recurrent vulvovaginal candidiasis. The manifestations of vulvovaginal candidiasis are often painful and uncomfortable and can include intense itching, irritation, vaginal discharge and dysuria [1], in addition to some issues related to social burden. Infectious vaginitis appears as a result of a violation of the normal flora of the vagina, which suppresses the immune response of the host and pathogenic microorganisms entering the vagina [2].

Although there is an expansion in the development of vaginal healthcare product, many of them didn't attain the desired patient acceptability as well as required biological safety and effectiveness. Most of the recorded problems are related to discontinuing use due to the low patient acceptability, which in turn increases the recurrences [3]. Therefore, there is a continuous need for safe, efficacious and well-tolerated regimens that reduce the incidence of vaginal infections over prolonged periods.

Oral and intravaginal metronidazole (MTZ) are used, in an average dose of $500 \mathrm{mg}$ daily, for the treatment of vaginal candidiasis [4]. Many reports focus on the utility of vaginal delivery of antimicrobial drugs compared to oral therapy due to several advantages including higher localized drug concentration and lower systemic exposure [3, 5-7]. This may be especially important in the case of pregnant patients. Vaginitis seems to be a complex infection, and very often, it is not possible to identify only one causative agent due to a mixed infection. In such cases, a multi-functional treatment seems to be crucial [2].

Ethnomedicine is concerned with the use of natural oils in medicine due to their multiple biological functions comprising: antiinflammatory, anti-oxidant, anti-microbial, cytoprotective and antiaging properties [8-10], in addition to its great economic interest and multiple nutritional and pharmaceutical values together with their safety. Coconut oil (CO) is obtained from Cocos nucifera Linn (Palmae) [11] and is present in the form of a white paste below $25{ }^{\circ} \mathrm{C}$; this can provide an advantage for the preparation of vaginal pessaries. Early studies suggest that medium-chain fatty acids (MCFA), commonly found in tropical oils such as coconut oil (CO), are bactericidal for gram-positive and gram-negative bacteria, fungi, protozoa, and viruses $[12,13]$. Some studies have reported the efficacy of CO against Candida albicans $[14,15]$. CO consists of $99 \%$ triglycerides with free fatty acids (less than $0.2 \%$ ) [16], in addition to lauric acid (C12), caprylic (C8) and capric acids (C10) [16], these components have been reported to exhibit an antimicrobial activity [17]. A proposed mechanism for the effects of CO suggests that membrane lipids are solubilized as the CO fatty acids integrate into the membrane. Electron microscopy demonstrates the disruption of bacterial cell membrane when exposed to CO fatty acids with subsequent lysis of the bacteria [12].

According to Gatefossé, suppocire ${ }^{\circledR}$ and ovucire ${ }^{\circledR}$ products are hard fat semi-synthetic bases produced by esterification of fatty acids. Their composition consists of a mixture of C12-C18 triglycerides. They can be used for vaginal and rectal delivery and have many advantages being inert, compatible with a wide variety of active ingredients, chemically stable, tolerance to the mucosa, safe and non-irritant (www. gatefosse. com).

Vaginal health care products must be sufficiently acceptable, accessible and easy to use so that they are used correctly and consistently; this is necessary to eradicate the vaginal infection. In 2004, the International Working Group for Microbicides (IWGM) published a list of "essential characteristics for a topical microbicide"[18], these are: effective, safe and easy to use.

This study aimed to improve the use, delivery and effect of metronidazole (MTZ) for the treatment of recurrent vaginal infections. Different lipids were used to prepare MTZ pessaries, 
namely, suppocire NA (SNA), suppocire NAIS (SIS), suppocire AM (SAM) and ovucire WL (OWL). These lipids were used alone or in conjunction with coconut oil (CO), which is a natural component with many beneficial effects and low side effects, in addition to its availability and low cost. The prepared pessaries were compared based on different physicochemical properties; then the selected pessaries were subjected to microbiological studies in experimental animals followed by histopathological examination and preliminary clinical investigation.

\section{MATERIALS AND METHODS}

\section{Materials}

Metronidazole was purchased from El-Nasr chemical company, Egypt. Suppocire NA15 (SNA), suppocire NAIS10 (SIS), suppocire AM (SAM) and ovucire WL 2944 (OWL) was kindly supplied from Gattefosseé établissements, France. Pure edible coconut oil (CO) which solidifies below $25{ }^{\circ} \mathrm{C}$, and melts at around $30{ }^{\circ} \mathrm{C}$ to form a liquid, was purchased from Hemani Int., Karachi, Pakistan. Sabouraud dextrose agar was purchased from Oxoid, England; its typical composition $(\mathrm{g} / \mathrm{l})$ is mycological peptone 10.0 ; glucose 40.0 ; agar 15.0, pH 5.6 \pm 02 . Sabouraud liquid medium was purchased from Oxoid, England. Candida albicans was isolated from the vaginal swab of a patient with vaginal candidiasis who had received no antifungal therapy. All other chemicals used in the study were of analytical grade and were obtained from the El-Nasr Company for Pharmaceutical Chemicals (Cairo, Egypt).

\section{Formulation of MTZ-loaded pessaries}

Metronidazole (MTZ) pessaries, each containing $500 \mathrm{mg}$ of the drug, were formulated using the fusion method. Briefly, the lipid was melted first using a water bath then coconut oil was added (in the case of the pessaries formed from lipid/coconut oil in a ratio of 1:1 $\mathrm{w} / \mathrm{w}$ ). MTZ was then added to the melted base under gentle stirring to ensure complete mixing and to enhance cooling. The mass was poured into a metal mould just before congealing. After solidification at room temperature, the prepared pessaries were packed in tightly closed containers and placed in a refrigerator. The composition of the different formulae is listed in table 1.

\section{Physical evaluation of MTZ-loaded pessaries}

\section{Weight variation}

The average weight was calculated by weighing 6 pessaries individually and the percentage deviation from the mean was determined.

\section{Disintegration time}

The test was performed in buffer $\mathrm{pH} 4.5$ at $37 \pm 0.5^{\circ} \mathrm{C}$ using Erweka tablet disintegration apparatus, Germany [19]. The disintegration time was recorded as soon as the pessaries placed in the basket either completely melt or dissolve according to the British Pharmacopoeia [20].

\section{Hardness}

This test was designed to measure the brittleness and fragility of the pessaries. The resistance of the prepared pessaries was evaluated using Erweka hardness tester (Germany) [19] under the effect of increasing pressure at room temperature. The force $\left(\mathrm{Kg} / \mathrm{cm}^{2}\right)$ required for the deformation and breaking of the pessaries was calculated.

\section{Melting range}

The test was carried out using the capillary method [21] in melting point apparatus. A straight capillary tube, 8 to $10 \mathrm{~cm}$ in length and 1 to $1.2 \mathrm{~mm}$ in internal diameter, opened at both ends was used. One end of the tube was dipped into the suppository bases, and gently sufficient amount was packed to fill $1 \mathrm{~cm}$ of the column (no previous melting of the sample was done to avoid changing any stable modification to unstable one). The capillary tube was then placed in the apparatus attached to a thermometer. The melting range was recorded when the contents of the capillary tube started to melt.

\section{In vitro release}

The in vitro dissolution studies were carried out using a USP rotating basket apparatus (Hanson Research Corporation, United States). The pessaries were placed in the baskets which were rotated at $50 \mathrm{rpm}$ in $500 \mathrm{ml}$ phosphate buffer $\mathrm{pH} 4.5$ maintained at $37 \pm 0.5^{\circ} \mathrm{C}$ [3]. An aliquot of $5 \mathrm{ml}$ of the solution was withdrawn at predetermined time intervals and replaced by fresh dissolution medium. The withdrawn samples were analyzed for MTZ content spectrophotometrically at a $\lambda \max$ of $318 \mathrm{~nm}$. Blank experiments were run in parallel to avoid any interaction.

Some release parameters were calculated to compare between investigated formulae [22], these comprised:

Release efficiency (RE) which is defined as the area under the release curve (AUC) at time $t$, calculated using the trapezoidal rule.

Mean release time (MRT) which is a measure of the release or dissolution rate; the higher the MRT, the slower is the release rate $[23,6]$.

Both, the MRT and RE, are model independent approaches which allow evaluating the drug release [24].

Also, kinetics of drug release was determined by finding the best fit of the release data to distinct models: zero-order and Higuchi order.

\section{$\mathrm{X}$-ray diffraction}

The X-ray powder diffraction patterns of MTZ, coconut oil, ovucire WL 2944 (OWL), and the selected preparation were recorded on a Diano X-ray diffractometer (Scintag Inc., USA). Samples were irradiated using Ni-filtered, CuK $\alpha$ radiation at a voltage of $45 \mathrm{kV}$, and a $9 \mathrm{~mA}$ current. The scanning rate employed was $1^{\circ} \mathrm{min}^{-1}$ over $0^{\circ}$ to $90^{\circ}$ diffraction angle $(2 \theta)$ range.

\section{Assessment of the antimicrobial activity}

The antimicrobial activity was assessed using the inhibition zone test $[3,25]$. The agar medium was prepared by dissolving $65 \mathrm{~g}$ of sabouraud dextrose agar powder per liter of distilled water and, then autoclaved at $121{ }^{\circ} \mathrm{C}$ for $20 \mathrm{~min}$. A turbid 48-h culture of $C$. albicans prepared in $200 \mathrm{ml}$ liquid Sabouraud medium; turbidity was adjusted to contain approximately $10^{5} \mathrm{cells} / \mathrm{ml}$ then poured into sterilized Petri plates under laminar air flow, allowed to solidify and then 4 uniform grooves were cut into the solidified media in each plate using a sterilized test tube. The MTZ-loaded pessaries (containing $20 \mathrm{mg}$ drug) [26], P4 and P8, were suspended in $10 \mathrm{ml}$ phosphate buffer $\mathrm{pH} 4.5$ and shaken at room temperature at $50 \mathrm{rpm}$. At different time intervals (15, 30, 60 and $120 \mathrm{~min})$, a sample of $2 \mathrm{ml}$ was withdrawn and filled into the different done cups using sterilized syringes, under laminar air flow. The withdrawn samples were replenished with fresh one [3]. Plates were covered with lids and incubated at $32{ }^{\circ} \mathrm{C}$ for $40 \mathrm{~h}$. The zones of inhibition were examined and their diameters were measured after $40 \mathrm{~h}$ [27].

\section{In vivo studies}

\section{Animals}

Female Wistar rats weighing between 180 and $220 \mathrm{~g}$ (6-8 w old) were obtained from the animal house of the National Research Center, Cairo, Egypt. The animals were housed in plastic cages and kept in a conditioned atmosphere at $22 \pm 3{ }^{\circ} \mathrm{C}$ and humidity $50-55 \%$ with $12 \mathrm{~h}$ light/dark cycles, they were fed standard pellet chow and were allowed free access to water. This study was conducted in accordance with the European Community guidelines and, was approved by animal care and use ethics committee of the faculty of Pharmacy Damanhour University (Ethical approval number118 PT4).

\section{Experiment}

In order to evaluate the antimicrobial activity, vaginal infection was induced using the rat vaginal model [28]. Animals were divided into four groups each containing 6 rats. At day 0 , the rats of the negative control group were injected $0.1 \mathrm{ml}$ normal saline intravaginally; while, all the other animals were inoculated intravaginally with $10^{7}$ yeast cells of $C$. albicans strain tested in $0.1 \mathrm{ml}$ of saline. The infected animals were divided into 2 groups, one group served as a positive control which didn't receive any treatment; and the other group was treated intravaginally with the selected pessaries (P8) loaded with 
$20 \mathrm{mg}$ of the drug [26], once daily for 6 consecutive days starting from the day following the induction of $C$. albicans infection (day 1 , $2,3,4,5$ and 6 ). At day 7 , the animals were sacrificed, and the vagina was excised and subjected to histopathological study.

\section{Histopathological examination}

Autopsy samples were taken from the vagina of different groups of female Wistar rats (negative control, positive control and P8) and were fixed with formalin for $24 \mathrm{~h}$. Washing was done in tap water then; serial dilutions of alcohol (methyl, ethyl and absolute ethyl) were used for dehydration. Specimens were cleared in xylene and embedded in paraffin at $56{ }^{\circ} \mathrm{C}$ in a hot air oven for $24 \mathrm{~h}$. Paraffin bees wax tissue blocks were prepared for sectioning at a thickness of $4 \mu \mathrm{m}$ by sledge microtome. The obtained tissue sections were collected on glass slides, deparaffinized, and then stained by hematoxylin and eosin stain for examination using the electric light microscope.

\section{Preliminary prospective clinical investigation}

This investigation is an outpatient trial obeying to the recommendations of the declaration of Helsinki and approved by the ethical committee of the faculty of Pharmacy Damanhour University (Approval Number 118 PT4). The questionnaire included healthy, normally menstruating women, suffering from recurrent vaginal infection and aged 25-50 y. The subjects provided their informed consent and were made clearly aware of their right to withdraw from the study at any time without giving any reason. The patients were instructed to insert the selected, designed pessaries (P8) or the market product (Amrizole ${ }^{\circledR}$ vaginal suppository), each containing $500 \mathrm{mg}$ MTZ, intravaginally at bedtime for 6 consecutive days, and then to respond to the questionnaire involving the symptoms they were suffering from [29]: itching, burning, vulvovaginal erythema and oedema, and vaginal secretion. The symptom severity was scored $0-3$, where $0=$ absent, $1=$ mild, $2=$ intense, $3=$ very intense. At the end of the treatment, treatment efficacy was assessed and categorized as $0=$ no efficacy; 1 = moderate efficacy; 2 = good efficacy. In addition, a questionnaire related to the likeability of the inserted pessaries was included in the study, the score was 0-2 starting from 0: not liked, 1: moderately liked, 2 : highly liked, the parameters included were the odor, ease of use, and non-irritancy.

\section{Statistical analysis}

Data were expressed as the meantthe standard deviation and were analyzed using one-way analysis of variance, followed by the least significant difference procedure using SPSS 10.0 software. Statistical differences are yielding $p<0.05$ were considered significant.

\section{RESULTS AND DISCUSSION}

\section{Physical evaluation of the MTZ-loaded pessaries \\ Weight variation}

Table 1 is listing the weight of the prepared pessaries, all of them showed uniform weight and low weight variation.

\section{Melting point}

As seen in table 1, the melting points of the lipid/CO-based pessaries were lower than the corresponding lipid-based ones; this is due to the addition of $\mathrm{CO}$ which has a melting point lower than that of the lipids under investigation. This can provide more privilege by allowing a better dispersion and spreading of the pessary in the vaginal lumen to cover the vaginal wall and permit a better contact with it, hence, better absorption and effect. A previous study reported that in the case of suppositories with lipid bases, lower melting point bases had demonstrated better drug release [30]. Also, rapid melting of the pessary upon application can decrease the risk of its expulsion out of the vaginal lumen.

\section{Hardness}

The values of the hardness of the prepared pessaries are listed in table 1 . All of them are in the range of 2.30 to $3.00 \mathrm{Kg} / \mathrm{cm}^{2}$, which is an indication of the ability of differently formulated pessaries to withstand pressure during handling, shipping and insertion.

\section{Disintegration time}

The disintegration time of the prepared pessaries are listed in table 1. All of them were in the range of 4.10-7.00 min with P1 and P8 attaining the highest and lowest values, respectively. The listed values run in parallel with those of the melting point because a higher melting point of a lipid base results in higher disintegration time. All the lipid/CO-based pessaries had longer disintegration time than the corresponding lipid-based one. A rapid disintegration can be beneficial as it leads to a rapid spreadability of the pessary in the vagina after application, which results in a rapid drug release and effect. This also can decrease the possibility of the discharge of the pessaries out of the vagina after application.

From the results displayed above, lipid/CO-based pessaries were selected for further investigations rather than a lipid-based suppository. The better physical properties of the former can allow better use and drug delivery into the vagina, in addition to the intrinsic anti-microbial properties of $\mathrm{CO}$; all these factors can permit for a better effect and higher patient compliance.

Table 1: Composition and physical properties of the MTZ-loaded pessaries

\begin{tabular}{|c|c|c|c|c|c|}
\hline Pessary & Composition & Melting range & Melting range $\left({ }^{0} \mathrm{C}\right)$ & Hardness $\left(\mathrm{kg} / \mathrm{cm}^{2}\right)$ & Disintegration time (min) \\
\hline P1 & SIS & $2.10 \pm 0.20$ & $38-40$ & $3.00 \pm 0.19$ & $7.00 \pm 0.25$ \\
\hline P2 & SAM & $2.20 \pm 0.10$ & $35-37$ & $2.90 \pm 0.17$ & $6.50 \pm 0.35$ \\
\hline P3 & SNA & $2.01 \pm 0.30$ & $35-37$ & $2.90 \pm 0.15$ & $6.30 \pm 0.40$ \\
\hline $\mathrm{P} 4$ & OWL & $2.10 \pm 0.10$ & $34-35$ & $2.50 \pm 0.10$ & $5.30 \pm 0.35$ \\
\hline P5 & SIS/CO (1:1) & $2.05 \pm 0.11$ & $36-37$ & $2.82 \pm 0.02$ & $5.00 \pm 0.25$ \\
\hline P6 & SAM/CO $(1: 1)$ & $2.21 \pm 0.17$ & $34-36$ & $2.60 \pm 0.03$ & $4.45 \pm 0.25$ \\
\hline P7 & SNA/CO (1:1) & $2.02 \pm 0.20$ & $34-35$ & $2.55 \pm 0.01$ & $4.30 \pm 0.30$ \\
\hline P8 & OWL/CO (1:1) & $2.05 \pm 0.24$ & $33-34$ & $2.30 \pm 0.02$ & $4.10 \pm 0.15$ \\
\hline
\end{tabular}

Lipids used were: suppocire NA (SNA), suppocire NAIS (SIS), suppocire AM (SAM) and ovucire WL 2944 (OWL), coconut oil (CO), metronidazole (MTZ), The values represent the mean $\pm S D(n=6)$

Table 2: Release parameters: release efficiency (RE), mean release time (MRT) and release kinetics

\begin{tabular}{lllll}
\hline Pessaries & MRT (min) & Release $\mathbf{~ R}^{2} \mathbf{)}$ & RE & \\
\cline { 3 - 4 } & & Zero-order & 0.98 & $21.81 \pm 2.62$ \\
P1 & $68.67 \pm 2.41$ & 0.99 & 0.98 & $28.43 \pm 2.66$ \\
P2 & $70.54 \pm 5.12$ & 0.99 & 0.97 & $29.31 \pm 2.11$ \\
P3 & $67.50 \pm 4.29$ & 0.99 & 0.99 & $53.51 \pm 4.80$ \\
P4 & $50.22 \pm 3.90$ & 0.97 & 0.91 & $26.94 \pm 2.36$ \\
P5 & $63.29 \pm 5.82$ & 0.99 & 0.98 & $37.13 \pm 3.99$ \\
P6 & $58.13 \pm 5.12$ & 0.98 & 0.92 & $34.63 \pm 3.51$ \\
P7 & $66.73 \pm 5.96$ & 0.92 & 0.96 & $65.62 \pm 5.62$ \\
P8 & $37.97 \pm 4.12$ & & & \\
\hline
\end{tabular}

The values represent the mean $\pm \operatorname{SD}(n=3)$ 


\section{In vitro release studies}

Release profiles are displayed in fig. 1 and release parameters (MRT and RE) are listed in table 2. Based on the presented data, it is clear that the lipid/CO-based pessaries attained a significantly higher and faster drug release than the corresponding lipid-based pessaries (P1 vs $\mathrm{P} 5, \mathrm{P} 2$ vs $\mathrm{P} 6, \mathrm{P} 3$ vs $\mathrm{P} 7$ and $\mathrm{P} 4$ vs $\mathrm{P} 8$ ). In the case of C0/lipid combination, a less ordered lipid matrix can be obtained by mixing lipid-oil ingredients [9], the drug molecules can be arranged in between the fatty acid chains or within the lipid layers, and also, in the imperfections of the lipid matrix, this can allow a better drug escape out of the lipid matrix forming the pessaries, upon contact with the external release media. Also, it is clear that P8 $(\mathrm{OWL} / \mathrm{CO}-$ based pessaries) attained the highest drug release compared with the other lipid/CO-based pessaries. This may be due to the differences in the structure of the different lipids which certainly affect drug release. A previous study has reported that the difference in the chain length and complexity, molecular arrangement and viscosity of the lipids led to a difference in the physicochemical properties [31]. The drug release profiles were expected from the results of the melting point and disintegration time of the prepared bases, a lower melting point, and more rapid disintegration allow for a faster drug release. Therefore, P8 was selected for the following investigations.

Regarding the best fitting kinetic model (table 2), all the prepared pessaries were found to follow a zero-order kinetic model, except for P8 which followed a Higuchian model.

\section{X-rays diffraction}

Fig. 2 is showing the X-rays diffraction pattern of the selected formula and each of the components alone. The diffractogram of the crystalline drug is showing well-defined, and sharp peaks at different positions, the most obvious appear at about $12.15^{\circ}, 12.30$ ${ }^{\circ}$, and $27.31^{\circ}$. CO shows a sharp crystalline peak at about $5.40^{\circ}$ and another broad amorphous peak at about $21.08^{\circ}$.

OWL shows different sharp peaks at about $4.66^{\circ}, 16.65^{\circ}, 19.08^{\circ}$ and $21.29^{\circ}$. While, the diffractogram of the selected pessary (P8) doesn't show any clear peak, which can point out to the complete fusion and coalition of the ingredients, and the presence of the drug in an amorphous form.

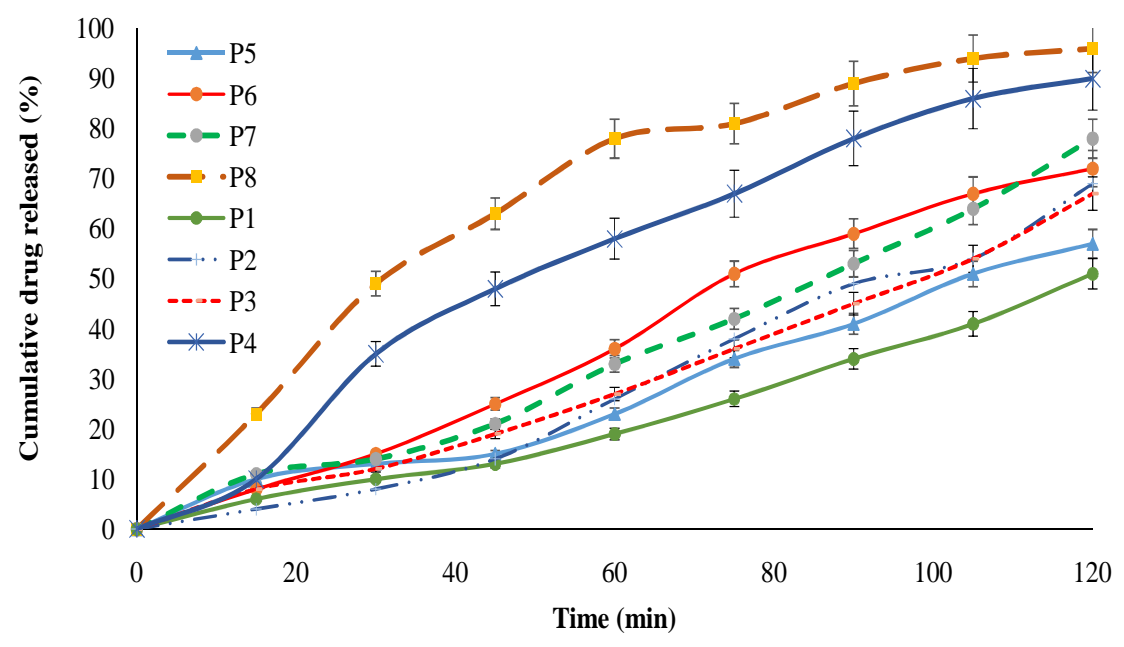

Fig. 1: In vitro release profiles of MTZ from the prepared pessaries, the values represent the mean $\pm \operatorname{SD}(n=3)$
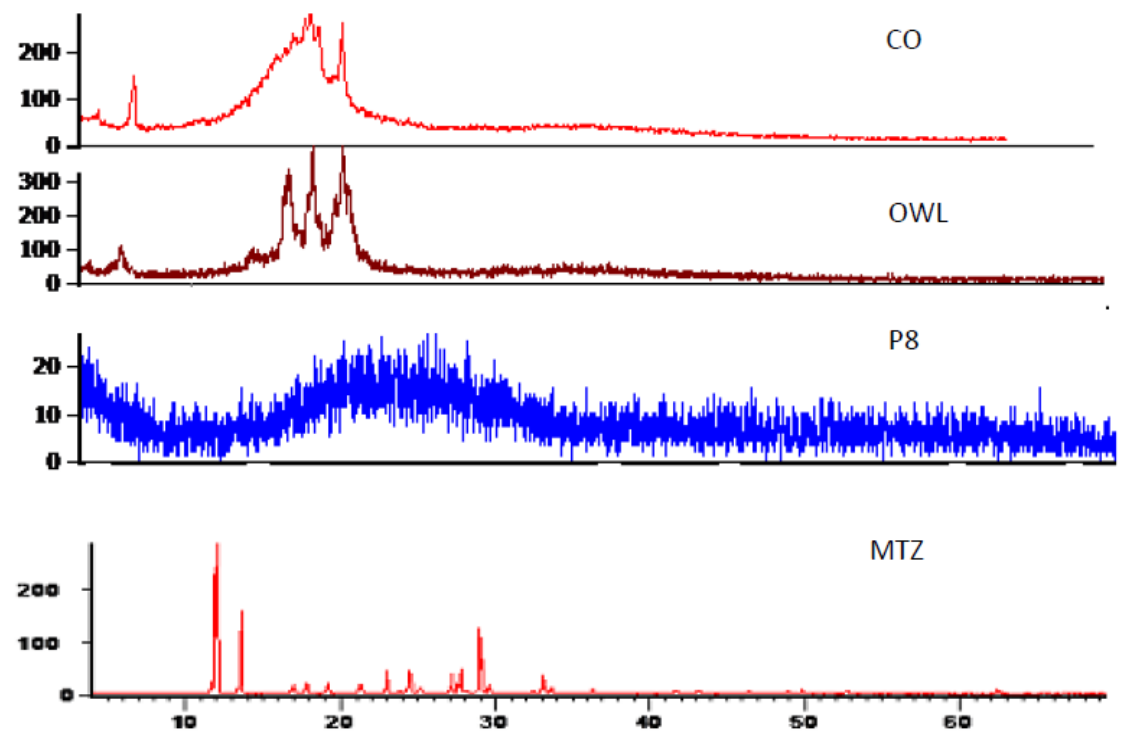

2-Theta

Fig. 2: X-ray patterns of metronidazole (MTZ), coconut oil (CO), ovucire WL (OWL) and the selected pessary (P8) 


\section{Assessment of the antimicrobial activity}

To validate the usefulness of the addition of $\mathrm{CO}$ to prepare the pessaries, the anti-microbial effectiveness of the MTZ-loaded OWL/CO-based pessary (P8) was compared with an MTZ-loaded OWL-based pessary (P4). Fig. 3 is a bar chart showing the diameter of the inhibition zones, at different time intervals, in the case of P4 and P8. Fig. 4 is presenting the photographs of the agar plates with the zones of inhibition of the fungal growth induced by P8 in comparison with those induced by $\mathrm{P} 4$, at different time intervals. At all time intervals, it was clear that the combination between MTZ and CO improved the anti-mycotic effect of the MTZ-loaded pessaries $(\mathrm{p}<0.05)$. This was expected due to the previous reports confirming the anti-microbial effect of the oil against Candida albicans $[14,15]$, which can potentiate the effect of MTZ. An additional advantage for the CO/lipid combination is the faster drug release from the base as discussed above in the in vitro release study. In addition, the results displayed above showed that the OWL/CO-based pessaries had a lower melting point and faster disintegration time than the OWL-based ones; this may allow a better dispersion of the pessary in the media and permit a more rapid and complete drug release. Another issue is that, upon use, lipid/CO base can have a softer texture than that of the lipid alone, which can promote its dispersion and spreadability, this can present an additional privilege in the case of in vivo use by the formation of a film on the vaginal wall.

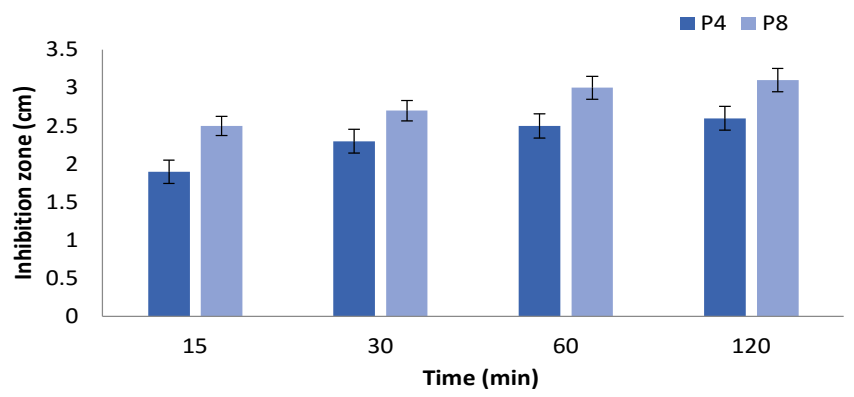

Fig. 3: A bar chart showing the diameter of the inhibition zones at different time intervals in the case of the OWL/CO-based pessary (P8) in comparison with the OWL-based pessary $(P 4)$, the values represent the mean $\pm S D(n=4)$
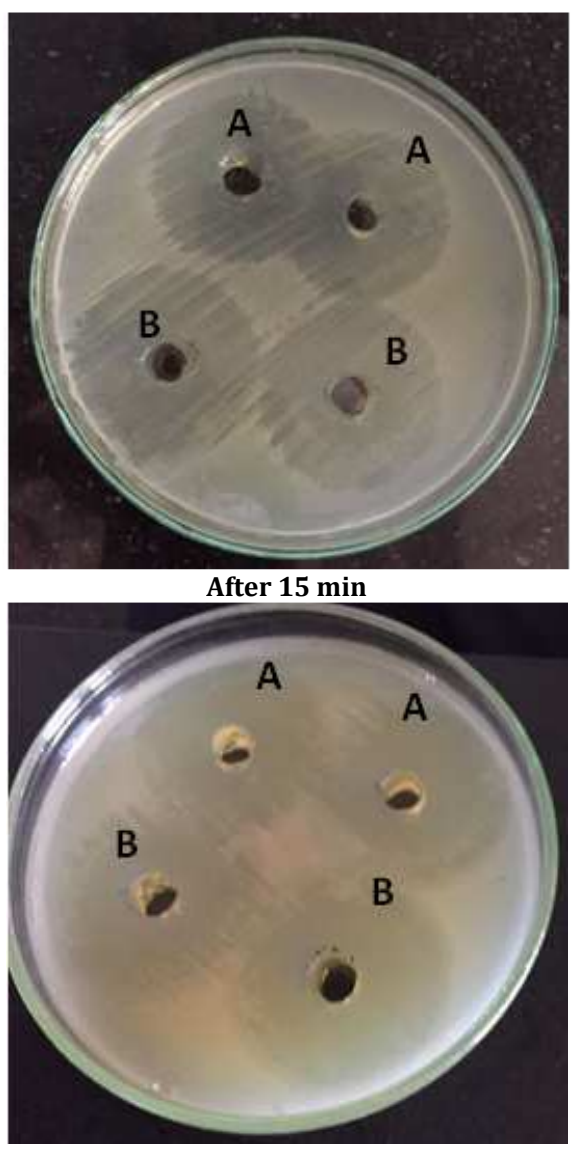

After 60 min

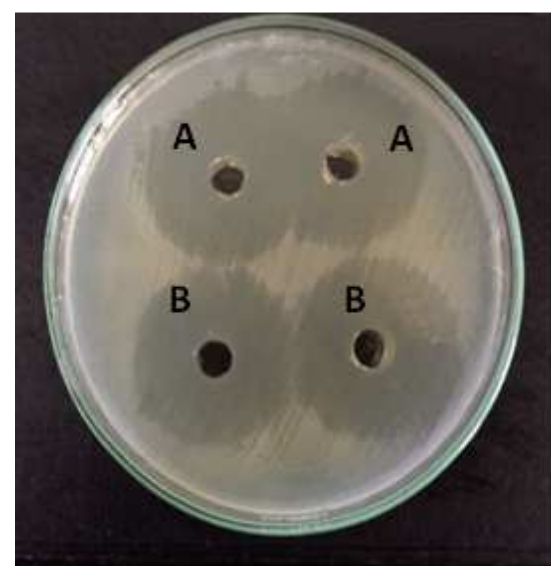

After $30 \mathrm{~min}$

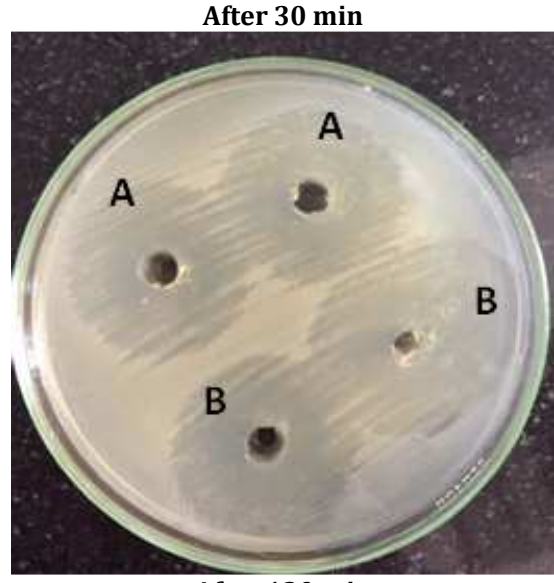

After 120 min

Fig. 4: Zones of inhibition of the fungal growth induced by OWL/CO-based pessary (P8) in comparison with the OWL-based pessary (P4) at different time intervals. A: P4, B: P8 


\section{In vivo study}

\section{Visual examination}

The activity of the selected pessary (P8), in comparison with a negative control and a positive control group, was tested in female Wistar rats before investigations involving the female volunteer. Fig. 5 is showing the photographs of the vaginal orifice of the rats at the end of the experiment. It is clear that in the case of the positive control ( $C$ albicans-infected group) the vaginal orifice showed erythema, edema and dilatation due to infection and inflammation. All these symptoms were absent in the case of the group treated with P8, and the vagina looked almost normal and similar to that of the negative control. This proves the effectiveness of the designed pessary and its capability to combat $C$. albicans vaginal infection.

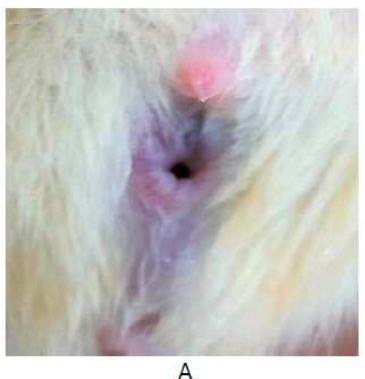

A

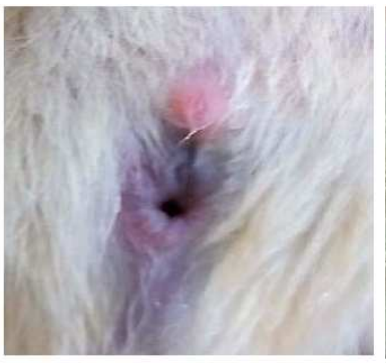

B

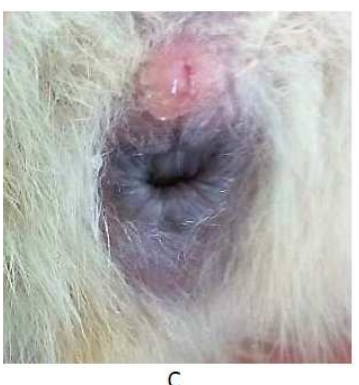

Fig. 5: Vaginal orifice of female wistar rats, (A) C. albicans-infected rats and treated with the selected preparation (P8), (B) Positive control (C. albicans-infected rats and received no treatment), (C) Negative control

\section{Histopathological study}

Fig. $6 \mathrm{~A}$ is showing the histological pattern of the vagina of the negative control group, there was no histopathological alteration, and the normal histological structure of the mucosal lining epithelium and the underlying muscular layer were recorded. While in fig. 6B, new blood capillaries (a)-resulting from the inflammation and edema-can be clearly detected. Also, there were mucosal hyperplasia and hyper stratification with cauliflower-like projections formation (b) associated with inflammatory cells infiltration in the underlying muscular layer. In addition, some inflammatory cells infiltration was detected in the muscular layer. These histological alterations resulted from $C$. albicans infection in the untreated group. In fig. 6C presenting the treated $C$. albicans-infected group, there was no histopathological alteration in the mucosal layer as well as the underlying musculature. The mucosal layer returned to its normal structure and the cauliflower-like projections disappeared; only a few inflammatory cells remained as a result of the defense mechanism. These results prove the in vivo efficacy, non-irritancy and mucosal tolerance of the suggested OWL/CO-based MTZ pessary.

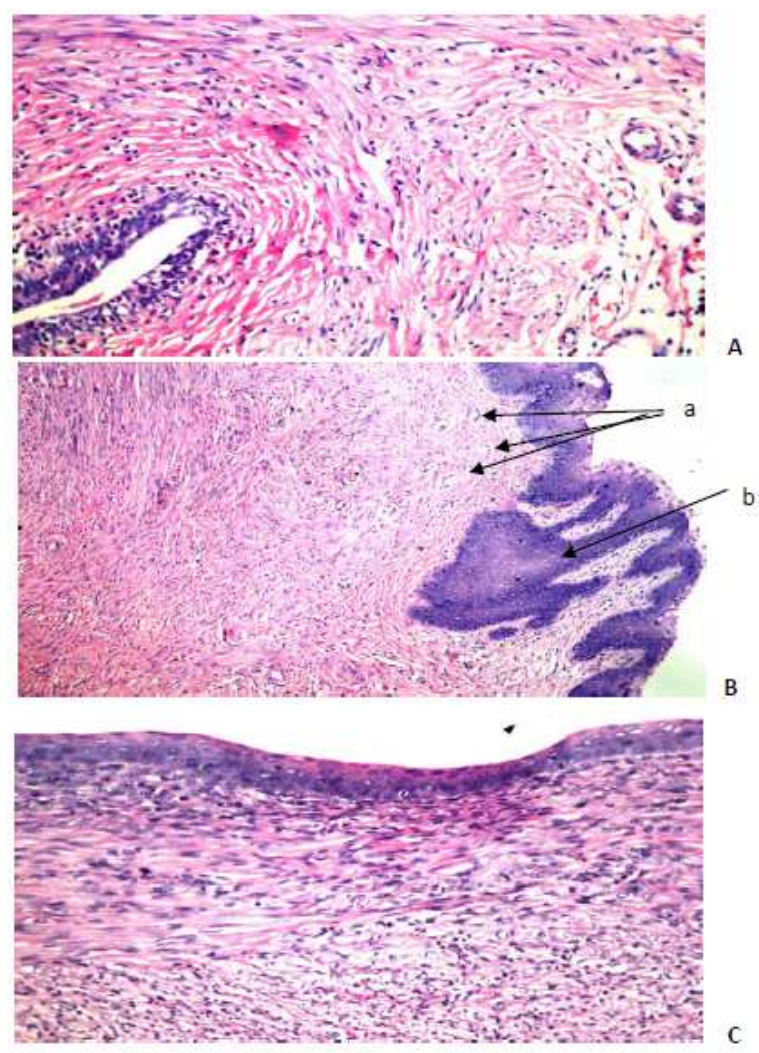

Fig. 6: Histopathological studies, A) Negative control, B) Positive control (C. albicans-infected rats and received no treatment), a) blood capillaries, b) cauliflower-like projection, C) Group treated with the selected preparation (C. albicans-infected rats and treated with P8) 


\section{Preliminary clinical investigation}

After the promising results are shown above about the high effectiveness of the suggested vaginal treatment, a preliminary questionnaire in women volunteers was run. For a vaginal preparation to be effective, it must be used properly and consistently. Some vaginal preparations show good results in vitro, but fail to do it in vivo due to the lack of acceptability of the preparation by the patients; therefore, this questionnaire was done to study the possibility of the application of the suggested vaginal pessary and to focus on the interplay between the biological and physicochemical properties of it. The selected, designed pessaries were compared with the market product, Amrizole ${ }^{\circledR}$ vaginal suppository.

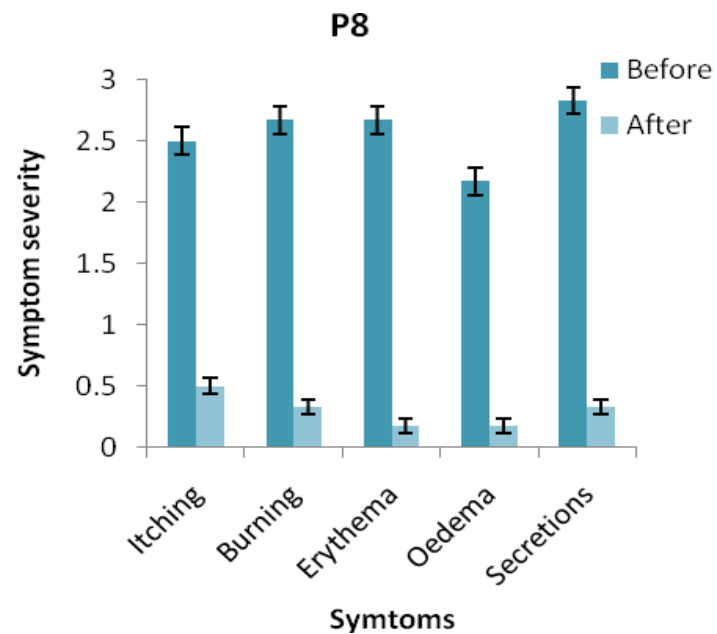

A
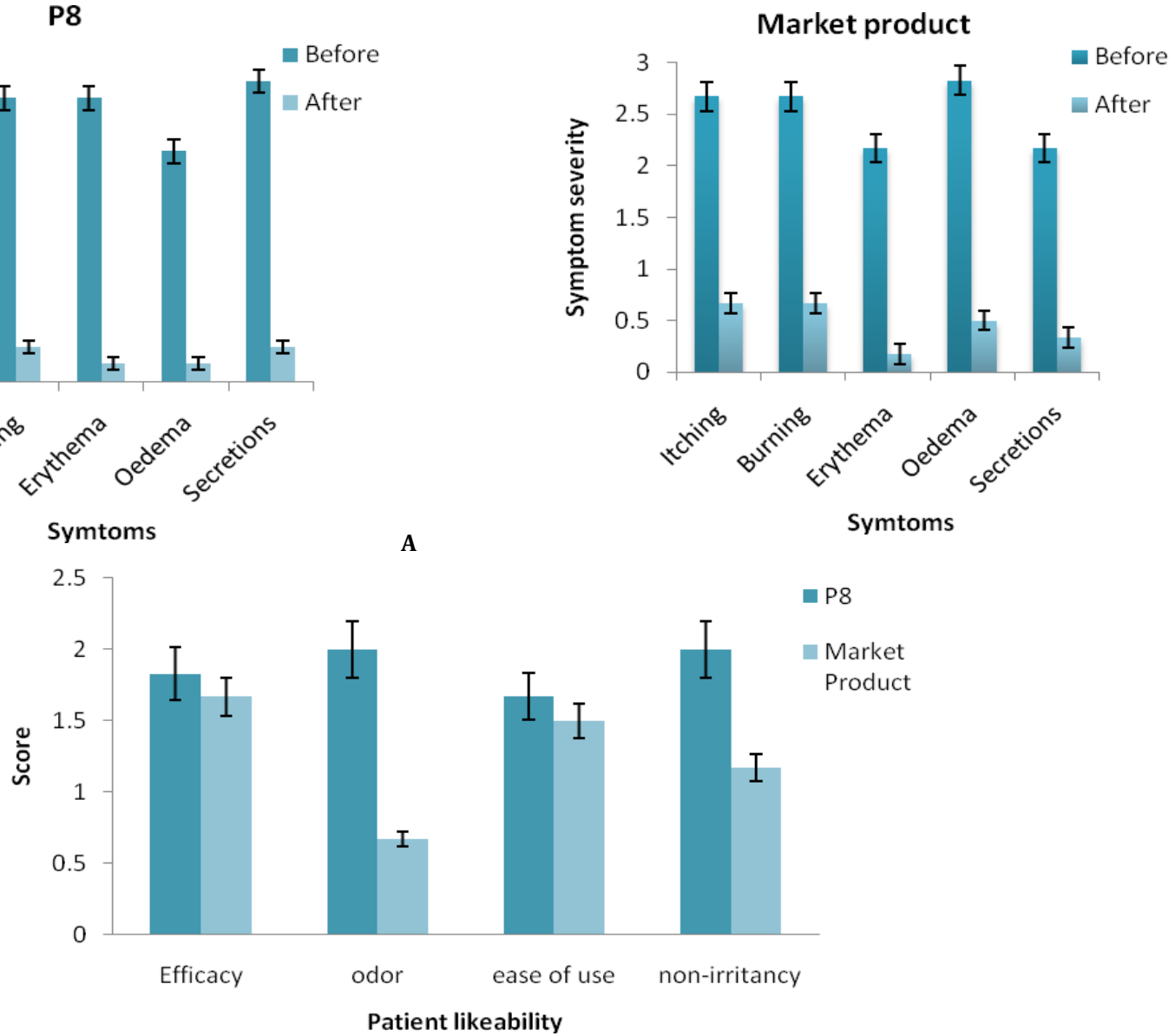

B

Fig. 7: Results reported by the questionnaire done in a group of women suffering from recurrent vaginal infection after receiving the intravaginal treatment, $A$ ) Symptoms before and after receiving the treatment, P8 or the market product (Amrizole ${ }^{\circledR}$ ). The symptom severity was scored $0-3$, where $0=$ absent, $1=$ mild, $2=$ intense, $3=$ very intense, $B$ ) Likeability of the MTZ-loaded pessaries used (P8) compared to the market product (Amrizole ${ }^{\circledR}$ ), the score was 0-2 starting from 0: not liked, 1: moderately liked, 2: highly liked, the values represent the mean $\pm S D(n=6)$

Fig. 7A shows the values of the severity score of the symptoms reported due to recurrent vaginal infection like itching, burning, vulvovaginal erythema and oedema, and vaginal secretion. The significant difference between symptoms severity, before and after treatment with P8 as well as Amrizole $\AA$, is clear $(\mathrm{p}<0.05)$. An improvement was reported in all the cases with a \% reduction in the severity of the symptoms equal to 80 , $87.64,93.63,92.17$ and 88.34 in the case of P8, and 74.91, 74.91, 92.17, 82.33 and 84.79 in the case of Amrizole $\AA$, for the itching, burning, vulvovaginal erythema and oedema, and vaginal secretion, respectively. The designed pessaries showed better results especially regarding the itching and burning sensation, this may be due to the intrinsic properties of $\mathrm{CO}$, in addition to the rapid disintegration and good dispersion and spreadability of P8 in the vaginal lumen allowing it to cover the vaginal wall, as explained above.

Acceptability is a term indicating the willingness to use a certain product [29], this factor is crucial for the successfulness of a vaginal preparation. The likeability of the preparation was evaluated in terms of its ease of use, odor, non-irritancy and efficacy. As shown in fig. 7B, for all the cases the designed preparation (P8) was moderately to highly liked with an average score of 1.83, 2, 1.67 and 2 for the efficacy, odor, ease of use and non-irritancy, respectively. While, in the case of Amrizole ${ }^{\circledR}$, the acceptability score was 1.67, 0.67, 1.50 and 1.17 for the efficacy, odor, ease of use and nonirritancy, respectively. The higher acceptability of the designed pessaries, especially regarding the odor, may be due to the pleasant odor of the $\mathrm{CO}$; this can provide an important advantage taking in consideration that vaginal infections are frequently accompanied with a bad odor which presents an additional disturbing symptom.

Based on the above listed results, we can point out to that the designed OWL/CO-based MTZ pessary obeys to the International Working Group for Microbicides (IWGM) guidelines about the necessity for a topical microbicide to be effective, safe and easy to use [18]. 


\section{CONCLUSION}

Balancing what people want (easy to use, pleasant, no side effects, low-cost, etc.) with what is feasible (physicochemical and formulation issues) will continue to be a challenge facing the development of a certain product. This study presents a simple, yet, attractive approach to prepare an anti-mycotic treatment to combat recurrent vaginal infection using a natural product. This approach was based on the use of Coconut oil to potentiate the anti-microbial effect of metronidazole and, to ameliorate the physicochemical properties of the designed pessaries. The in vitro as well as in vivo studies, histopathological examination and preliminary clinical investigations highlight the success of the suggested vaginal care product. In turn, this can decrease social burden and improve the quality of life of a large women population.

\section{ACKNOWLEDGMENT}

The authors would like to thank the Microbiology Department, Faculty of Pharmacy, Damanhour University, Egypt, for the help in the microbiological experiment.

\section{AUTHORS CONTRIBUTIONS}

All the author have contributed equally

\section{CONFLICT OF INTERESTS}

The authors declare that they have no conflict of interest

\section{REFERENCES}

1. Horowitz BJ, Giaquinta D, Ito S. Evolving pathogens in vulvovaginal candidiasis: implications for patient care. J Clin Pharmacol 1992;32:248-55.

2. Dolzhykova O, Maloshtan L, Yeriomenko R, Maloshtan V. Effect of the vaginal pessaries Melanizol ${ }^{\circledR}$ and Klimedeks $®$ on the glycogen level in the vaginal tissue of rats on the background of experimental vaginitis. Eur PMC 2017;66:117-26.

3. Kamel R, Abbas H. A multi-microcarrier of metronidazolebiopolymers complexes as a potential vaginal delivery system. Int J Polym Mater Polym Biomater 2018;67:236-45.

4. Bradshaw CS, Morton AN, Hocking J, Garland SM, Morris MB, Moss LM, et al. High recurrence rates of bacterial vaginosis over the course of $12 \mathrm{mo}$ after oral metronidazole therapy and factors associated with recurrence. J Infect Dis 2006;193:1478-86.

5. Sobel JD, Wiesenfeld HC, Martens M, Danna P, Hooton TM, Rompalo A, et al. Maintenance fluconazole therapy for recurrent vulvovaginal candidiasis. New England J Med 2004;351:876-83.

6. Kamel R. Study of the influence of selected variables on the preparation of prolonged release bioadhesive vaginal carbohydrate microspheres using an experimental design. J Drug Delivery Sci Tech 2013;23:247-54.

7. Khattab A, Ismail S. Formulation and evaluation of oxiconazole nitrate mucoadhesive nanoemulsion-based gel for treatment of fungal vaginal infection. Int J Pharm Pharm Sci 2016;8:33-40.

8. Tsai ML, Lin CC, Lin WC, Yang CH. Antimicrobial, antioxidant, and anti-inflammatory activities of essential oils from five selected herbs. Biosci Biotechnol Biochem 2011;75:1977-83.

9. Kamel R, Abbas H, Fayez A. Diosmin/essential oil combination for dermal photo-protection using a lipoid colloidal carrier. J Photochem Photobiol B 2017;170:49-57.

10. Sivapriya V, Ponnarmadha S, Azeezand NA, Sudarshanadeepa $V$. Novel nanocarriers for ethnopharmacological formulations. Int J Appl Pham 2018;10:26-30.

11. Santos SS, Lorenzoni A, Pegoraro NS, Denardi LB, Alves SH, Schaffazick SR, et al. Formulation and in vitro evaluation of coconut oil-core cationic nanocapsules intended for vaginal delivery of clotrimazole. Colloids Surf B 2014;116:270-6.

12. Kabara JJ, Swieczkowski DM, Conley AJ, Truant JP. Fatty acids and derivatives as antimicrobial agents. Antimicrob Agents Chemother 1972;2:23-8.

13. Skřivanova E, Marounek M, Benda V, Brezina P. Susceptibility of escherichia coli, salmonella sp and clostridium perfringens to organic acids and monolaurin. Veterinarni Medicina; 2006.

14. Bergsson G, Steingrímsson O, Thormar H. In vitro susceptibilities of neisseria gonorrhoeae to fatty acids and monoglycerides. Antimicrob Agents Chemother 1999;43:2790-2.

15. Ogbolu DO, Oni AA, Daini OA, Oloko A. In vitro antimicrobial properties of coconut oil on candida species in Ibadan, Nigeria. J Med Food 2007;10:384-7.

16. Marina A, Man YC, Nazimah S, Amin I. Chemical properties of virgin coconut oil. J Am Oil Chem Soc 2009;86:301-7.

17. Bergsson G, Arnfinnsson J, Steingrimsson O, Thormar H. In vitro killing of candida albicans by fatty acids and monoglycerides. Antimicrob Agents Chemother 2001;45:3209-12.

18. Lard Whiteford SL, Matecka D, O'Rear JJ, Yuen IS, Litterst C, Reichelderfer P. Recommendations for the nonclinical development of topical microbicides for prevention of HIV transmission: an update. J Acquired Immune Defic Syndr 2004;36:541-52.

19. Belniak P, Swiąder K, Szumiło M, Hyla A, Poleszak E. Comparison of physicochemical properties of suppositories containing starch hydrolysates. Saudi Pharma J 2017;25:365-9.

20. In British Pharmacopoeia: London; 1993. p. 159-60.

21. Abass H, Kamel R, Abdelbary A. Metronidazole bioadhesive vaginal suppositories: formulation, in vitro and in vivo evaluation. Int J Pharm Pharm Sci 2012;4:344-55.

22. Kamel R, Abbas H. PLGA-based monolithic filaments prepared by hot-melt extrusion: In vitro comparative study. Ann Pharm Françaises 2018;76:97-106.

23. Varshosaz J, Tavakoli N, Kheirolahi F. Use of hydrophilic natural gums in formulation of sustained-release matrix tablets of tramadol hydrochloride. AAPS PharmSciTech 2006;7:E24.

24. Kamel R, Abbas HA. Self-assembled carbohydrate hydrogels for prolonged pain management. Pharm Dev Technol 2013;18:990-1004.

25. Liu $\mathrm{Y}$, Zhang $\mathrm{X}$, Liu $\mathrm{Y}$, Jin $\mathrm{X}$, Fan $\mathrm{C}$, $\mathrm{Ye} \mathrm{H}$, et al. Bifunctionalization of a calcium phosphate-coated titanium surface with slow-release simvastatin and metronidazole to provide antibacterial activities and pro-osteodifferentiation capabilities. PloS One 2014;9:e97741.

26. Perioli L, Ambrogi V, Pagano C, Scuota S, Rossi C. FG90 chitosan as a new polymer for metronidazole mucoadhesive tablets for vaginal administration. Int J Pharm 2009;377:120-7.

27. Joseph M, Al-Hakami A, Assiry M, Jamil A, Assiry A, Shaker M, et al. In vitro anti-yeast activity of chloramphenicol: a preliminary report. J Mycol Med 2015;25:17-22.

28. De Bernardis F, Lorenzini R, Cassone A. Chapter 89-Rat Model of Candida Vaginal Infection A2-Zak, Oto, in Handbook of Animal Models of Infection. MA Sande. Editor. Academic Press: London; 1999. p. 735-40.

29. Woodsong C, Holt JDS. Acceptability and preferences for vaginal dosage forms intended for prevention of HIV or HIV and pregnancy. Adv Drug Delivery Rev 2015;92:146-54.

30. Bergren MS, Battle MM, Halsteadt GW, Theis DL. Investigation of the relationship between melting-related parameters and in vitro drug release from vaginal suppositories. J Pharm Biomed Anal 1989;7:549-61.

31. Kamel R, Mostafa DM. Rutin nanostructured lipid cosmeceutical preparation with sun protective potential. J Photochem Photobiol B 2015;153:59-66. 